\title{
Multiple-scattering production of lepton pairs in ultraperipheral heavy-ion collisions
}

\author{
Sevgi Karadağ ${ }^{*}$ and Mehmet Cem Güçlü ${ }^{\dagger}$ \\ Department of Physics, Istanbul Technical University, Istanbul, Turkey
}

(Received 11 March 2020; accepted 15 June 2020; published 6 July 2020)

\begin{abstract}
Ultra-relativistic peripheral collisions of heavy ions at the CERN Large Hadron Collider (LHC) can produce copies of numbers of lepton pairs via the two-photon process. Since the energies of the heavy ions are so high, multipair production cross sections of the light leptons, especially electrons, are quite large so that it is possible to measure them experimentally. To calculate the multipair production probabilities, first, we should have an impact parameter dependence cross section. We have obtained a well-behaved impact parameter dependence cross section and by using the Monte Carlo methods, we have calculated multipair production cross sections of electrons and muons in $\mathrm{Pb}-\mathrm{Pb}$ heavy-ion collisions at $\mathrm{LHC}$ energies. We have also used some experimental restrictions in our calculation to compare our findings with the experimental results.
\end{abstract}

DOI: 10.1103/PhysRevC.102.014904

\section{INTRODUCTION}

One of the main goals of the BNL Relativistic Heavy-Ion Collider (RHIC) and CERN Large Hadron Collider (LHC) is to investigate the quark-gluon plasma which is the confined state of the partonic matter. In central collisions of the heavy ions, dileptons are produced as a result of the quark-antiquark interaction (Drell-Yan process), and these dileptons interact only with electromagnetically with the particles $[1,2]$. The lepton-hadron interaction is very small hence they carry direct information from the first formation of space-time.

The ultraperipheral collisions of heavy ions are described by an impact parameter $b$ greater than the sum of the radius of the colliding nuclei. When two ultrarelativistic heavy ions pass near each other, the intense electromagnetic fields are very strong so that they pull lepton pairs from the vacuum. Lepton pairs coming from the quark-gluon plasma can be mixed with the electromagnetically produced lepton pairs. Therefore, it is extremely important to investigate the electromagnetic production of lepton pairs in detail.

From the pure science point of view, relativistic heavy-ion collisions provide an opportunity to study nonperturbative quantum electrodynamics (QED). In such collisions, colliding energies and electric charges are so high that the coherent electromagnetic production of lepton pairs using heavy ions is fundamentally different from other production mechanisms using light particles at high energies. In the heavy-ion case, the

\footnotetext{
*karadags@itu.edu.tr

$\dagger$ guclu@itu.edu.tr
}

Published by the American Physical Society under the terms of the Creative Commons Attribution 4.0 International license. Further distribution of this work must maintain attribution to the author $(s)$ and the published article's title, journal citation, and DOI. Funded by $S C O A P^{3}$. coupling constant is strongly enhanced due to the large charge. Nonetheless, the problem of reliable lowest order perturbative calculations for the lepton pair production has been used as input into design models for the RHIC and LHC.

We start with the quantum-field theory, and derive a classical field method based on the Feynman perturbation theory. In these calculations, we should be careful that the momentum transfer of the photons is much smaller than the momentum carried by the nuclei. This assumption is valid for the coherent production of lepton pairs through two-photon processes in relativistic heavy-ion collisions. By using these lowest-order diagrams, coupling lepton fields to classical electromagnetic fields have been evaluated exactly with the help of the Monte Carlo method and analytical techniques [3-6], and have been predicted cross sections consistent with the experiments for the lepton pair production.

On the other hand, by using the lowest-order perturbation theory to the production of electron-positron pairs with heavy ions at high energies and small impact parameters results in probabilities and cross sections which violate the unitarity $[1,7]$. It is therefore clear that low-order perturbative calculations alone are not adequate for smaller impact parameters at the RHIC and especially at the LHC energies. Therefore, higher-order terms must be included in the calculations.

In literature, electron-positron pair production from the vacuum is treated by applying the equivalent-photon approximation (EPA). In 1924, Fermi replaced the time-dependent electric field by an approximately equivalent spectrum of on-shell photons. The problem of the moving Coulomb field of the charged particles was done in the 1930s. Weizsacker and Williams modified this problem for the relativistic particles. In this approximation, the equivalent-photon flux created by the relativistic charged particle is obtained via a Fourier decomposition of the electromagnetic interaction $[7,8]$. Cross sections are calculated by folding the elementary, real two-photon cross section for the pair-production process using the equivalent-photon flux produced by each ion. The 
pair-production cross section is relatively easier to compute using the equivalent-photon approximation than by calculating the two-photon diagram. When we compare these two methods, the results for the total cross section are reasonably accurate provided the incident-particle Lorentz factor $\gamma$ is much greater than one and the energy transferred via the photon is much less than $\gamma$. However, the details of differential cross-sections, spectra, and impactparameter dependence differ, especially when complicated numerical cuts in the coordinates are applied in order to compare with experiments. Among the shortcomings of this approximation is an undetermined parameter which corresponds to the minimum impact parameter or the maximum momentum transfer, which makes it difficult to get specific results. As such, the method loses the applicability at impact parameters less than the Compton wavelength of the lepton $[9,10]$, which is the region of greatest interest for the study of nonperturbative effects. Reference [4] reviews the application of the equivalent-photon method in relativistic heavy-ion collisions.

The recent experiments at RHIC and LHC provided us with several data about electromagnetic production of lepton pairs from the ultraperipheral collisions of the heavy ions. The rapidity, invariant mass, and transverse momentum distributions are measured with the experimental cutoffs. In our calculations, we have estimated these observables with the experimental limits and compared them with the recent ALICE and ATLAS data for the electron-positron and muonantimuon pair production [11-18]. We have also predicted the electromagnetic production of double $e^{+} e^{-}$and $\mu^{+} \mu^{-}$pair productions.

\section{FORMALISM}

The cross sections for producing a final state $X$ in the equivalent-photon method are written as the integral over the "photon fluxes" of each of the ions times the on-shell two-photon to the $X$ cross section

$$
\sigma=\iint d \omega_{1} d \omega_{2} n_{A_{1}}\left(\omega_{1}\right) n_{A_{2}}\left(\omega_{2}\right) \sigma_{\gamma \gamma \rightarrow X}\left(\omega_{1} \omega_{2}\right)
$$

where $n(\omega)$ is the equivalent photon distribution, and $A$ is the colliding nuclei. The EPA is an approximation to the two-photon external field model that we studied in this article.

Figure 1 shows two heavy ions moving with relativistic velocities $u$ along the $z$ axis in opposite directions to each other. They undergo the Lorentz contraction due to their high relativistic velocities. The distance between the radii of the nuclei is shown by the impact parameter $b$. Throughout this paper, we use the natural units with $\hbar=c=m=e=1$.

Since the physical processes such as lepton-pair production or annihilation occur with the interaction of fields, the lepton pair production from the electromagnetic field is expressed by the interaction lagrangian density which depends on the classical four-vector potential $A^{\mu}$ :

$$
\mathcal{L}_{\text {int }}(x)=-\bar{\psi}(x) \gamma_{\mu} \psi(x) A^{\mu}(x)
$$

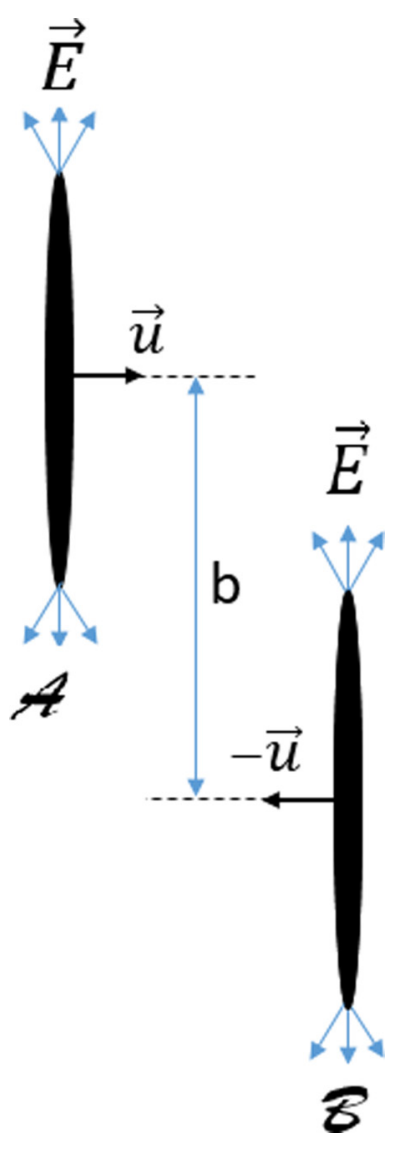

FIG. 1. Collision of two heavy-ions at relativistic velocities.

where $A^{\mu}$ can be written separately for two colliding nuclei $\mathcal{A}$ and $\mathcal{B}$

$$
A^{\mu}(q, b)=A_{\mathcal{A}}^{\mu}(q, b)+A_{\mathcal{B}}^{\mu}(q, b) .
$$

The four-vector potential in momentum space can be written as

$$
\begin{aligned}
A_{(\mathcal{A}, \mathcal{B})}^{0}(q)= & -8 \pi^{2} \gamma^{2}(Z e) G_{E}\left(q^{2}\right) f_{z}\left(q^{2}\right) \\
& \times \exp \left( \pm i \mathbf{q}_{\perp} \cdot \frac{\mathbf{b}}{2}\right) \frac{\delta\left(q_{0} \mp \beta q_{z}\right)}{q_{z}^{2}+\gamma_{\perp}^{2}}, \\
A_{(\mathcal{A}, \mathcal{B})}^{z}(q)= & \pm \beta A_{(\mathcal{A}, \mathcal{B})}^{0}(q), \\
\mathbf{A}_{(\mathcal{A}, \mathcal{B})}^{\perp}(q)= & 0,
\end{aligned}
$$

where $f_{z}\left(q^{2}\right)$ and $G_{E}\left(q^{2}\right)$ are the form factors. Form factors are crucial for the cross-section calculation especially for heavy lepton-pair production in electromagnetic collisions. In our calculations, we have used the Fermi (Woods-Saxon) distribution for the charge distribution of the nucleus,

$$
\rho(r)=\frac{\rho_{0}}{1+\exp \left(\frac{r-R}{a}\right)},
$$

where $R$ is the radius of the nucleus, and $a$ is a quantity related to the thickness of the nucleus shell [19]. Also, $\rho_{0}$ can be calculated by normalization.

Using the expressions given so far, the cross section depending on the impact parameter $b$ for the lepton pair 
(a)

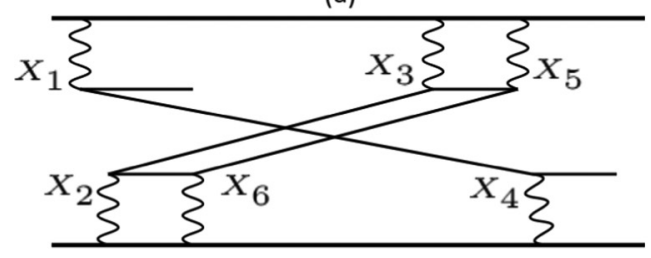

(b)

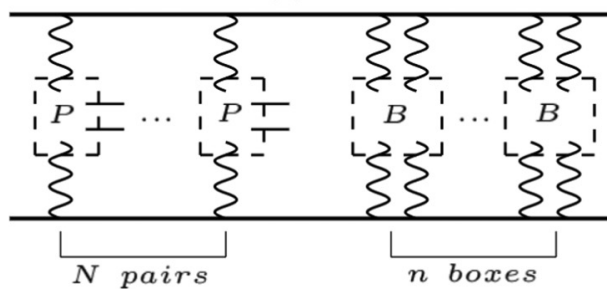

FIG. 2. (a) contains one pair creation and one box. All of the diagrams in (b) which we include are in the form of $N$ pair creations and $n$ boxes.

production can be written

$$
\sigma=\int d^{2} \mathbf{b} \sum_{k>0} \sum_{q<0}\left|\left\langle\chi_{k}^{(+)}|S| \chi_{q}^{(-)}\right\rangle\right|^{2}
$$

where the summation over the states $k$ is restricted to those above the Dirac sea, and the summation over the states $q$ is restricted to those occupied in the Dirac sea.

The result for $S_{\mathcal{A B}}$ is

$$
\begin{aligned}
\left\langle\chi_{k}^{(+)}\right. & \left.\left|S_{\mathcal{A B}}\right| \chi_{q}^{(-)}\right\rangle \\
= & \frac{i}{2 \beta} \int \frac{d^{2} \mathbf{p}_{\perp}}{(2 \pi)^{2}} \exp \left[i\left(\mathbf{p}_{\perp}-\frac{\left(\mathbf{k}_{\perp}+\mathbf{q}_{\perp}\right)}{2}\right) \mathbf{b}\right] \\
& \times F\left(\mathbf{k}_{\perp}-\mathbf{p}_{\perp} ; \omega_{\mathcal{A}}\right) F\left(\mathbf{p}_{\perp}-\mathbf{q}_{\perp} ; \omega_{\mathcal{B}}\right) \mathcal{T}_{k q}\left(\mathbf{p}_{\perp} ; \beta\right),
\end{aligned}
$$

where the functions $F(\mathbf{q}, \omega)$ and $\mathcal{T}_{k q}\left(\mathbf{p}_{\perp} ; \beta\right)$ can be written explicitly as

$$
\begin{aligned}
& F\left(\mathbf{k}_{\perp}-\mathbf{p}_{\perp} ; \omega_{\mathcal{A}}\right)=\frac{4 \pi Z \gamma^{2} \beta^{2}}{\omega_{\mathcal{A}}^{2}+\gamma^{2} \beta^{2}\left(\mathbf{k}_{\perp}-\mathbf{p}_{\perp}\right)^{2}}, \\
& F\left(\mathbf{p}_{\perp}-\mathbf{q}_{\perp} ; \omega_{\mathcal{A}}\right)=\frac{4 \pi Z \gamma^{2} \beta^{2}}{\omega_{\mathcal{B}}^{2}+\gamma^{2} \beta^{2}\left(\mathbf{p}_{\perp}-\mathbf{q}_{\perp}\right)^{2}},
\end{aligned}
$$

$$
\begin{aligned}
\mathcal{T}_{k q}\left(\mathbf{p}_{\perp} ; \beta\right) & \\
= & i \sum_{s} \sum_{\sigma_{p}}\left[E_{p}^{(s)}-\left(\frac{E_{k}^{(+)}+E_{q}^{(-)}}{2}\right)+\beta\left(\frac{k_{z}-p_{z}}{2}\right)\right]^{-1} \\
& \times\left\langle u_{\sigma_{k}}^{(+)}\left|\left(1-\beta \alpha_{z}\right)\right| u_{\sigma_{p}}^{(s)}\right\rangle\left\langle u_{\sigma_{p}}^{(s)}\left|\left(1+\beta \alpha_{z}\right)\right| u_{\sigma_{q}}^{(-)}\right\rangle .
\end{aligned}
$$

Here, $\left|\chi_{k}^{(+)}\right\rangle$refers to the positive-energy spinors, and $\left|\chi_{q}^{(-)}\right\rangle$ refers to the negative-energy spinors. $S$ is the scattering matrix and expressed as a series expansion. In our previous works, we have obtained exact cross-section expressions and tried to calculate it with Monte Carlo integration methods $[20,21]$. However, the Bessel function in this equation is rapidly oscillating, especially for large impact parameters, it makes it difficult to calculate the cross section numerically. On the other hand, an impact-parameter dependent cross section is very helpful for understanding the physical phenomena occurring in heavy-ion collisions. Therefore, to overcome this problem the cross-section expression can be divided into two parts:

$$
\frac{d \sigma}{d b}=\int_{0}^{\infty} q d q b J_{0}(q b) \mathcal{F}(q) .
$$

Here, $\mathcal{F}(q)$ is a nine-dimensional integral [Eq. (14)] that can be calculated with the Monte Carlo integration method [20].

When this expression is calculated for different values of $q$ with the Monte Carlo integration method, we can fit the results to a smooth function for $\mathcal{F}(q)$ :

$$
\begin{aligned}
\mathcal{F}(q)= & \frac{\pi}{8 \beta^{2}} \sum_{\sigma_{k}} \sum_{\sigma_{q}} \int_{0}^{2 \pi} d \phi_{q} \int \frac{d k_{z} d q_{z} d^{2} k_{\perp} d^{2} K d^{2} Q}{(2 \pi)^{10}}\left\{F\left(\frac{\mathbf{Q}-\mathbf{q}}{2} ; \omega_{\mathcal{A}}\right) F\left(-\mathbf{K} ; \omega_{\mathcal{B}}\right) \mathcal{T}_{k q}\left(\mathbf{k}_{\perp}-\frac{\mathbf{Q}-\mathbf{q}}{2} ; \beta\right)\right. \\
& \left.+F\left(\frac{\mathbf{Q}-\mathbf{q}}{2} ; \omega_{\mathcal{A}}\right) F\left(-\mathbf{K} ; \omega_{\mathcal{B}}\right) \mathcal{T}_{k q}\left(\mathbf{k}_{\perp}-\mathbf{K} ;-\beta\right)\right\}\left\{F\left(\frac{\mathbf{Q}+\mathbf{q}}{2} ; \omega_{\mathcal{A}}\right) F\left(-\mathbf{q}-\mathbf{K} ; \omega_{\mathcal{B}}\right) \mathcal{T}_{k q}\left(\mathbf{k}_{\perp}-\frac{\mathbf{Q}+\mathbf{q}}{2} ; \beta\right)\right. \\
& \left.+F\left(\frac{\mathbf{Q}+\mathbf{q}}{2} ; \omega_{\mathcal{A}}\right) F\left(-\mathbf{q}-\mathbf{K} ; \omega_{\mathcal{B}}\right) \mathcal{T}_{k q}\left(\mathbf{k}_{\perp}+\mathbf{q}-\mathbf{K} ;-\beta\right)\right\} .
\end{aligned}
$$

Here, $\omega_{\mathcal{A}}$ and $\omega_{\mathcal{B}}$ are the frequencies of the virtual photons:

$$
\begin{aligned}
& \omega_{\mathcal{A}}=\frac{E_{q}^{(-)}-E_{k}^{(+)}+\beta\left(q_{z}-k_{z}\right)}{2}, \\
& \omega_{\mathcal{B}}=\frac{E_{q}^{(-)}-E_{k}^{(+)}-\beta\left(q_{z}-k_{z}\right)}{2} .
\end{aligned}
$$

The transformations used in this equation are

$$
\begin{aligned}
\mathbf{Q} & =2 \mathbf{k}_{\perp}-\mathbf{p}_{\perp}-\mathbf{p}_{\perp}^{\prime}, \\
\mathbf{q} & =\mathbf{p}_{\perp}-\mathbf{p}_{\perp}^{\prime}, \\
\mathbf{K} & =-\mathbf{p}_{\perp}+\mathbf{q}_{\perp} .
\end{aligned}
$$


(a)

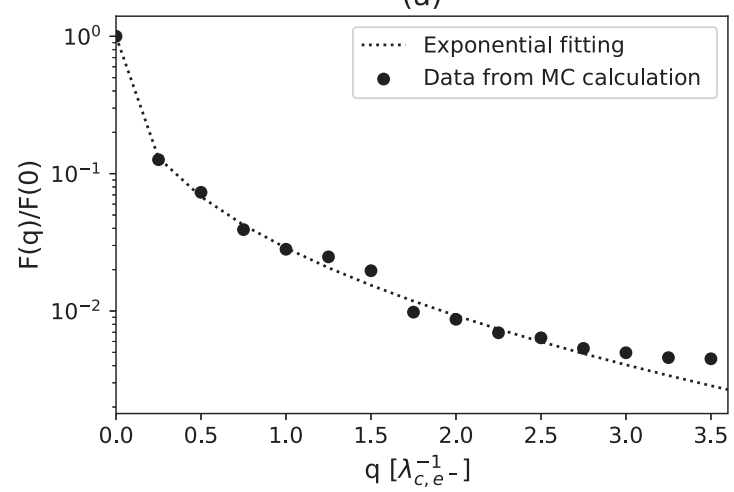

(b)

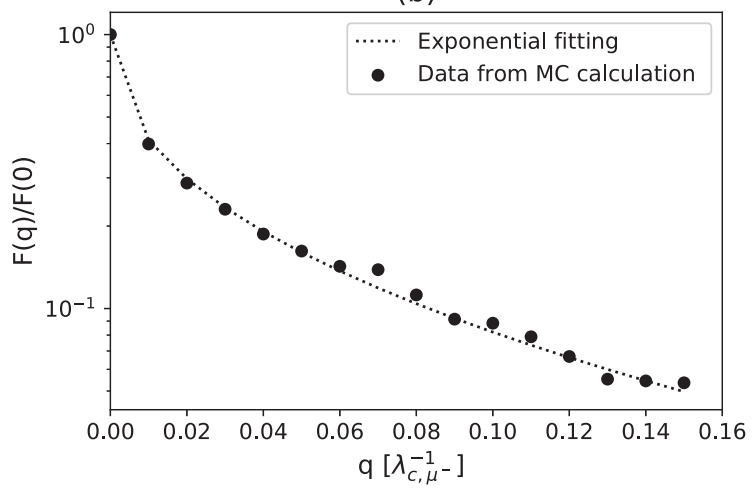

FIG. 3. The change of $\mathcal{F}(q) / \mathcal{F}(0)$ depended on $q$ variable for both (a) $e^{+} e^{-}$and (b) $\mu^{+} \mu^{-}$pairs production from $\mathrm{Pb}-\mathrm{Pb}$ collisions at LHC energies. (Here, $\lambda_{c}$ represents the Compton wavelengths of electron and muon.)

We have done all these calculations for different energies for producing electron and muon pairs, and have shown the results in Fig. 3. In our previous works, these smooth fits were generally in the form of $\approx e^{-a q}$. However, when we improved our calculations, we have found that the most appropriate fits should be in the form of

$$
\mathcal{F}(q)=\mathcal{F}(0) e^{-a_{1} q^{a_{2}}},
$$

where $\mathcal{F}(0)$ gives the total cross section $\sigma_{T}$ at $q=0$. The parameters $a_{1}$ and $a_{2}$ are obtained from the suitable fit functions.

To calculate the differential cross section, we can insert the fit function obtained in Eq. (17) into Eq. (13)

$$
\frac{d \sigma}{d b}=\mathcal{F}(0) \int_{0}^{\infty} q d q b J_{0}(q b) e^{-a_{1} q^{a_{2}}}
$$

By using the differential cross section, one can calculate the total cross section, multipair production probabilities, and the other observables.

\section{MULTIPAIR CREATION}

There are important reasons to understand multiple-pair production. When we increase the colliding energies, one-pair production cross sections become very large, and multipair production probability has also an important contribution to the observed cross sections. For high charged ions such as $\mathrm{Au}, \mathrm{Pb}$, and for very high energies, such as at the LHC, the lowest order perturbative calculations are not sufficient to calculate the probabilities of lepton pairs creation. Therefore, we need higher-order Feynman diagrams to evaluate the cross sections. However, it is very cumbersome to get expressions and to evaluate them. On the other hand, we can make some approximations and obtain sufficient results. In literature and in our previous works, details of the approximations are shown in a clear manner [4,6,9]. As an approximation, only the Fermion loops which have exactly two vertices attached to each of the ions are included. As an example, in Fig. 2(a) , one pair creation and one "box" are shown. To calculate the $N$ pair creations, we should include $N$ pair creations and $n$ boxes as shown in Fig. 2(b). We did not include the additional interactions such as the final state interactions of a created lepton with one of the ions. Therefore, when we calculate the sum of all this diagrams, we obtain the Poisson form for the multipair production

$$
P_{N}(b)=\frac{\mathcal{P}(b)^{N} e^{-\mathcal{P}(b)}}{N !},
$$

(a)

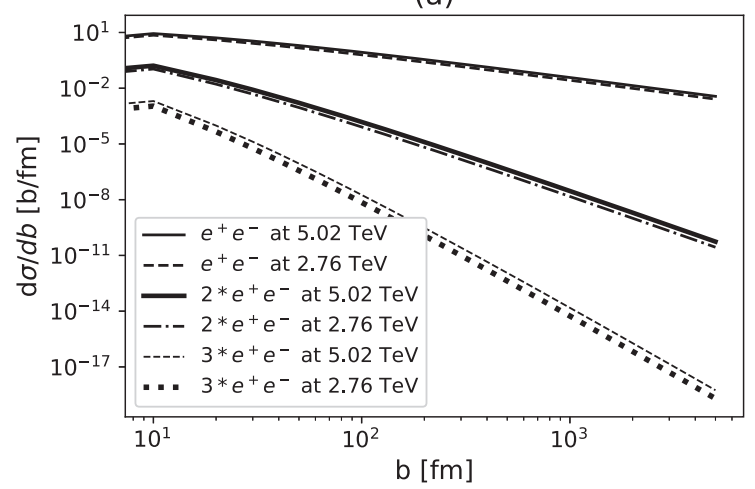

(b)

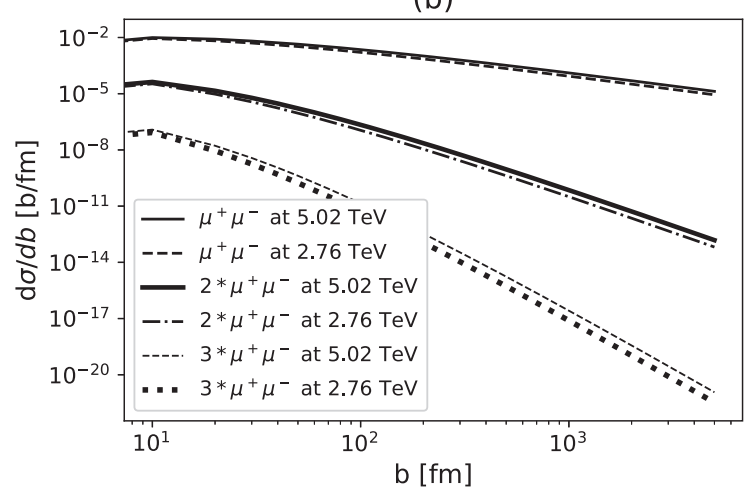

FIG. 4. The change of the cross section of (a) electron and (b) muon for single, double, and triple pairs production from $\mathrm{Pb}-\mathrm{Pb}$ collisions dependent on the impact parameter $b$ at LHC energies, $\sqrt{s_{N N}}=2.76$ and $5.02 \mathrm{TeV}$. 
(a)

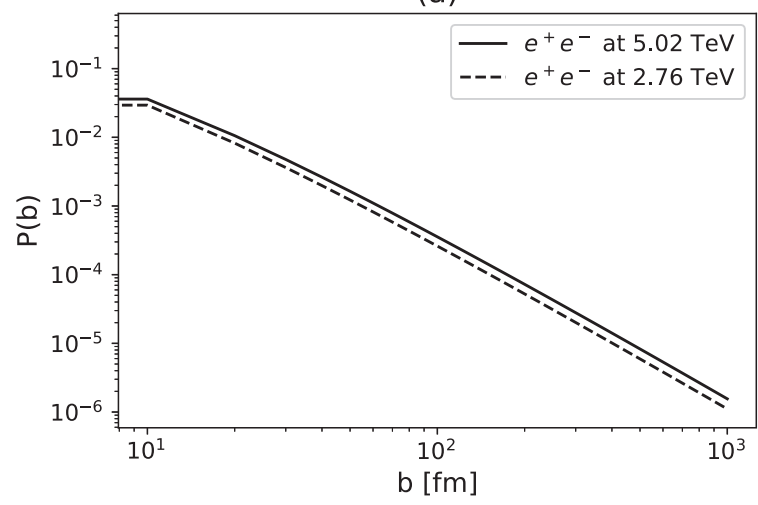

(b)

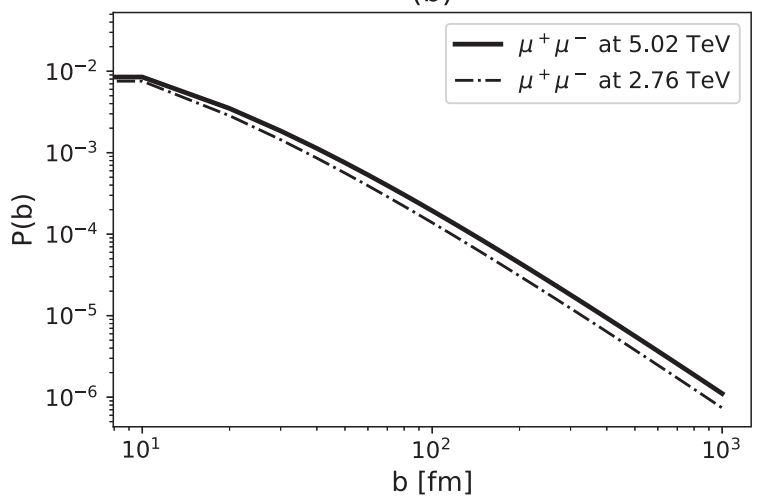

FIG. 5. The probabilities for (a) $e^{+} e^{-}$and (b) $\mu^{+} \mu^{-}$pairs production from $\mathrm{Pb}-\mathrm{Pb}$ collision at $\mathrm{LHC}$ energies.

where $N$ is the number of pairs, and $\mathcal{P}(b)$ is the probability of producing a single pair from the second-order Feynman diagrams

$$
\mathcal{P}(b)=\frac{1}{2 \pi b} \frac{d \sigma}{d b} .
$$

As we discussed above, this probability is not accurate for large values of charges and energies of the colliding ions. However, by obtaining the Poisson form for the multipair production, the perturbative result for the production of a single pair is modified by the exponential factor. In order to obtain $N$ pair cross section, we just need to integrate the $N$-pair probability $P_{N}(b)[5,9,20,21]$,

$$
\sigma_{N_{\text {pair }}}=\int d^{2} b P_{N}(b)
$$

\section{RESULTS AND DISCUSSIONS}

In the two photon-external field model that we study here, the most important task is to evaluate Eq. (14) numerically. We have done this by using the Monte Carlo integration method. We have used sufficiently large random numbers to evaluate it accurately.

TABLE I. $\sigma_{T}$ values for the production $e^{+} e^{-}$and $\mu^{+} \mu^{-}$pairs from $\mathrm{Pb}-\mathrm{Pb}$ collisions at $\mathrm{LHC}$ energies. The first group shows the single lepton-pair production, while the second group shows double lepton-pair production. The last group is for triple pair production.

\begin{tabular}{lcc}
\hline \hline Lepton pairs & $\begin{array}{c}2.76 \mathrm{TeV} \\
\text { (barn) }\end{array}$ & $\begin{array}{c}5.02 \mathrm{TeV} \\
\text { (barn) }\end{array}$ \\
\hline$e^{+} e^{-}$ & $1.67 \times 10^{5}$ & $2.17 \times 10^{5}$ \\
$\mu^{+} \mu^{-}$ & 1.67 & 2.30 \\
$e^{ \pm}-e^{ \pm}$ & $3.29 \times 10^{3}$ & $4.26 \times 10^{3}$ \\
$\mu^{ \pm}-\mu^{ \pm}$ & $1.72 \times 10^{-3}$ & $2.22 \times 10^{-3}$ \\
$e^{ \pm}-\mu^{ \pm}$ & $1.61 \times 10^{-1}$ & $2.07 \times 10^{-1}$ \\
$e^{ \pm}-e^{ \pm}-e^{ \pm}$ & $5.76 \times 10^{2}$ & $7.42 \times 10^{2}$ \\
$\mu^{ \pm}-\mu^{ \pm}-\mu^{ \pm}$ & $7.73 \times 10^{-6}$ & $11.7 \times 10^{-6}$ \\
\hline \hline
\end{tabular}

Figures 3(a) and 3(b) show the values of $\mathcal{F}(q) / \mathcal{F}(0)$ for each values of $q$ in $\mathrm{Pb}-\mathrm{Pb}$ collisions at $\mathrm{LHC}$ for electron and muon pairs productions, respectively. A smooth function is then fit to the calculated numbers. By using this fit function, we can calculate the impact parameter dependent cross sections and probabilities of the producing lepton pairs. These calculations are shown in Figs. 4(a) and 4(b) for electron and muon pairs, respectively. In these plots, the colliding energies of the $\mathrm{Pb}$ ions are considered as $\sqrt{s_{N N}}=2.76$ and $5.02 \mathrm{TeV}$ and we have plotted the single pair, double pairs, and triple pairs differential cross sections. After obtaining the differential cross sections, from Eqs. (19) and (20), we can calculate the probabilities of $N$ pair productions. These probabilities are plotted in Figs. 5(a) and 5(b) as a function of impact parameter for the above energies.

The calculated values of total cross sections $\sigma_{T}$ for single, double, and triple lepton-pairs production are shown in Table I. It is clear that, when the colliding energies increase, total cross sections increase as a result of this. In our calculations, we have used the Fermi (Woods-Saxon) distribution for the charge distribution of the nucleus, Eq. (7). Since the Compton wavelength of the muon is comparable with the size of the nucleus, form factors are very crucial in the calculations. For simplicity, we have tabulated the ratio between the multipair total cross-section values in Table II. Although, electron single-pair production is the dominant process, the double and triple pairs production mechanism

TABLE II. The ratio between $\sigma_{T}$ values of single, double, and triple pairs production from $\mathrm{Pb}-\mathrm{Pb}$ collisions. The first group shows electron pairs while the second group shows muon pairs.

\begin{tabular}{lcc}
\hline \hline Lepton pairs & $2.76 \mathrm{TeV}$ & $5.02 \mathrm{TeV}$ \\
\hline$\sigma_{1 p}^{e^{-}} / \sigma_{2 p}^{e^{-}}$ & $0.51 \times 10^{2}$ & $0.51 \times 10^{2}$ \\
$\sigma_{1 p}^{e^{-}} / \sigma_{3 p}^{e^{-}}$ & $0.29 \times 10^{3}$ & $0.29 \times 10^{3}$ \\
$\sigma_{2 p}^{e^{-}} / \sigma_{3 p}^{e^{-}}$ & $0.57 \times 10^{1}$ & $0.57 \times 10^{1}$ \\
$\sigma_{1 p}^{\mu^{-}} / \sigma_{2 p}^{\mu^{-}}$ & $0.97 \times 10^{3}$ & $1.04 \times 10^{3}$ \\
$\sigma_{1 p}^{\mu^{-}} / \sigma_{3 p}^{\mu^{-}}$ & $0.22 \times 10^{6}$ & $0.20 \times 10^{6}$ \\
$\sigma_{2 p}^{\mu^{-}} / \sigma_{3 p}^{\mu^{-}}$ & $0.22 \times 10^{3}$ & $0.19 \times 10^{3}$ \\
\hline \hline
\end{tabular}


(a)

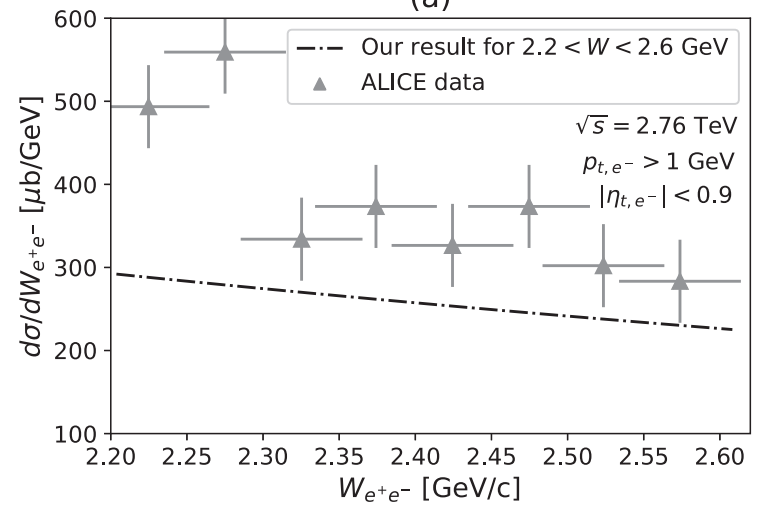

(b)

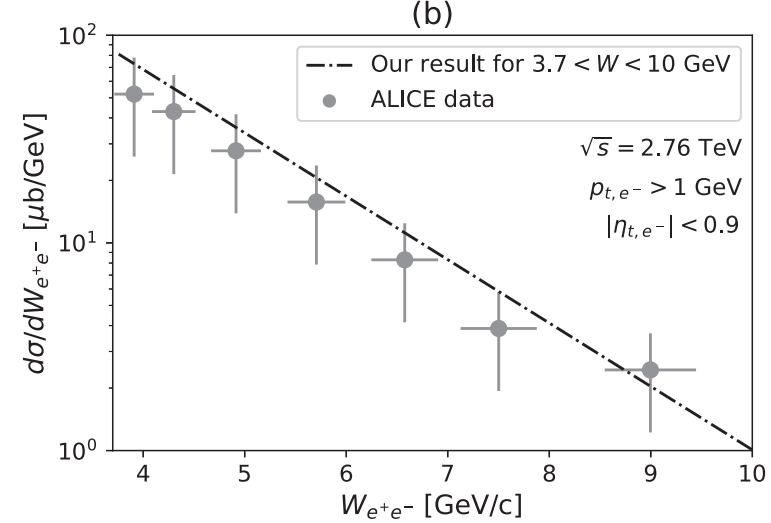

FIG. 6. The (a) low and (b) high invariant-mass distribution of $e^{+} e^{-}$pair in $\mathrm{Pb}-\mathrm{Pb}$ heavy-ion collisions at $\mathrm{LHC}\left(\sqrt{s_{N N}}=2.76 \mathrm{TeV}\right)$ energy together with the ALICE experiment data [12].

also has a significant contribution to the total pair production process. On the other hand, due to the higher mass, the gaps between the ratios of muon multipair cross sections are higher compared to electron-pair productions.

So far, we have not used any experimental cuts in our calculations on parameters, such as rapidity, transverse momentum, and invariant mass of the produced leptons. However, due to some technical difficulties, experimentally, cross sections are measured for limited values of the parameters. Therefore, in order to compare our calculations with the experimental results, we have also restricted our calculations within the experimental limits.

In the ALICE experiment, the kinematic restrictions for the measured $e^{+} e^{-}$pair production from $\mathrm{Pb}-\mathrm{Pb}$ collisions at $\sqrt{s_{N N}}=2.76 \mathrm{TeV}$ are $|y|<0.9$ for rapidity of all electrons and pairs, $2.2>W_{e^{+} e^{-}}>10 \mathrm{GeV}$ for invariant mass, and $p_{t, e^{-}}>1 \mathrm{GeV}$ for transverse momentum. We have applied these restrictions to our calculations, and shown our results in Figs. 6(a) and 6(b) for both low invariant-mass range $2.2<W_{e^{+} e^{-}}<2.6 \mathrm{GeV}$ and high invariant-mass range $3.7<$ $W_{e^{+} e^{-}}<10 \mathrm{GeV}$, respectively. Then, we integrated these curves over invariant-mass ranges to find total cross-section values, and showed the results in Table III. While our result slightly overestimates the experimental result for the high invariant-mass range, we obtained a lower value for the low invariant-mass range. On the other hand, STARLIGHT calculations [22] are slightly lower than the experimental results.
In the ATLAS experiment, the kinematic restrictions for the production of a $\mu^{+} \mu^{-}$pair in $\mathrm{Pb}-\mathrm{Pb}$ collisions at $\sqrt{s_{N N}}=$ $5.02 \mathrm{TeV}$ are $|y|<2.4$ for rapidity, $W_{\mu^{+} \mu^{-}}>10 \mathrm{GeV}$ for invariant mass, and $p_{t, \mu^{-}}>4 \mathrm{GeV}$ for transverse momentum. We have applied these restrictions in our calculation, and our results for the differential cross section are shown as a function of invariant mass in Fig. 7. In this figure, the area under the curve gives us the total cross-section value with all the restrictions mentioned above. In Table III, we have tabulated our results for the muon pair production and compared them with the experimental result and STARLIGHT calculation. Our result is slightly lower than the experimental result and STARLIGHT calculation. Similar calculations have been done by using EPA method and the results can be found in Refs. [23-30].

In conclusion, we have compared our calculations with the ATLAS and ALICE experimental results. Although the measured physical quantities have been obtained with many restrictions, hence only the small portions of the produced leptons are observed, and they are not inconsistent with our calculations. This means that the two-photon external field model is useful for predicting the electromagnetic lepton pair production mechanism. Most importantly, accurate calculations of the impact parameter dependent cross section help us in many other predictions such as multi-lepton-pair production. Our calculations predict that probabilities of two or three pair production of electron and muon are quite high, and likely to be measured. There was an attempt to measure the two-pair

TABLE III. First line shows the experimental and calculated results of total cross sections at the rapidity range $|y|<2.4$ for $\mu^{+} \mu^{-}$pair at $\sqrt{s_{N N}}=5.02 \mathrm{TeV}$ (ATLAS). The other lines show the results for $e^{+} e^{-}$pair at the rapidity range $|y|<0.9$ at $\sqrt{s_{N N}}=2.76 \mathrm{TeV}$ (ALICE).

\begin{tabular}{lccc}
\hline \hline Lepton pair & $\begin{array}{c}\text { Our result } \\
(\mu \mathrm{b})\end{array}$ & $\begin{array}{c}\text { LHC [11,12] } \\
(\mu \mathrm{b})\end{array}$ & $\begin{array}{c}\text { STARLIGHT [22] } \\
(\mu \mathrm{b})\end{array}$ \\
\hline$\mu^{+} \mu^{-}$ & 25 & $32.2 \pm 0.3(\mathrm{stat})_{-3.4}^{+4.0}$ (sys) & $31.64 \pm 0.04($ stat $)$ \\
$e^{+} e^{-}$ & 117 & $91 \pm 10(\mathrm{stat})_{-8}^{+11}(\mathrm{sys})$ & 77 \\
$(3.7<W<10)$ & 103 & $154 \pm 11(\mathrm{stat})_{-11}^{+17}(\mathrm{sys})$ & 128 \\
$e^{+} e^{-}$ & & & \\
$(2.2<W<2.6)$ & & & \\
\hline \hline
\end{tabular}




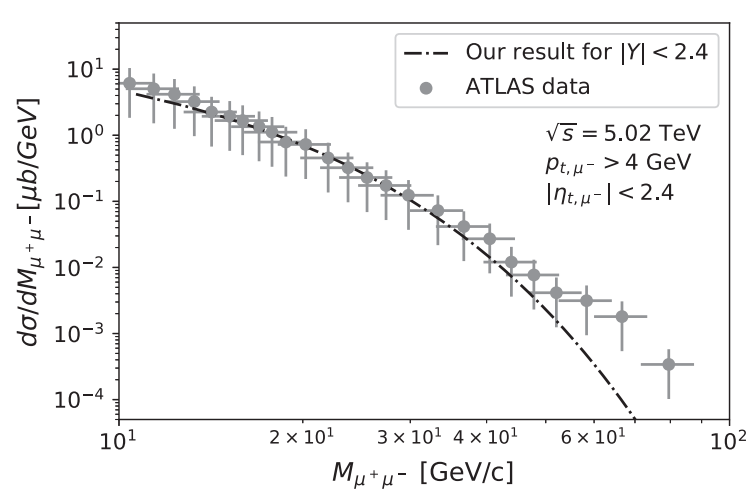

FIG. 7. The invariant mass distribution of $\mu^{+} \mu^{-}$pair in $\mathrm{Pb}-\mathrm{Pb}$ heavy-ion collisions at LHC $\left(\sqrt{s_{N N}}=5.02 \mathrm{TeV}\right)$ energy together with the ATLAS experiment data [11].

production cross sections [31,32] for $\mathrm{S}+\mathrm{Au}$ at $200 \mathrm{~A} \mathrm{GeV}$ and $\mathrm{Pb}+\mathrm{Au}$ at $160 \mathrm{~A} \mathrm{GeV}$ collisions. Since the colliding energies are small compared to the LHC energies, multipair production was not experimentally detected. However, theoretical calculations of the multipair production cross sections become high with the increase of the colliding energies, therefore, it may help to measure the multipair productions of the leptons. All these calculations and measurements help us to better understand the QED in a strong field.

Also, we have made some predictions for multipair production applying different experimental cuts on transverse
TABLE IV. $\sigma_{T}$ values for the multipair production from $\mathrm{Pb}-\mathrm{Pb}$ collisions at $5.5 \mathrm{TeV}$ for different cuts on individual transverse momentums and rapidities of produced pairs. The first column shows the double electron-pair production, while the second column shows the double muon-pair production. The last group is for simultaneous production of electron and muon pairs.

\begin{tabular}{lrrr}
\hline \hline Cuts & $e^{+} e^{-} e^{+} e^{-}$ & $\mu^{+} \mu^{-} \mu^{+} \mu^{-}$ & $e^{+} e^{-} \mu^{+} \mu^{-}$ \\
\hline$p_{t_{l}}>0.2 \mathrm{GeV}$ & $70.1 \mu \mathrm{b}$ & $64.9 \mu \mathrm{b}$ & $66 \mu \mathrm{b}$ \\
$p_{t_{l}}>0.2 \mathrm{GeV},\left|y_{l}\right|<2.5$ & $13.44 \mu \mathrm{b}$ & $9.3 \mu \mathrm{b}$ & $9.38 \mu \mathrm{b}$ \\
$p_{t_{l}}>0.2 \mathrm{GeV},\left|y_{l}\right|<1$ & $0.538 \mu \mathrm{b}$ & $0.453 \mu \mathrm{b}$ & $0.476 \mu \mathrm{b}$ \\
$p_{t_{l}}>0.3 \mathrm{GeV},\left|y_{l}\right|<4.9$ & $13.9 \mu \mathrm{b}$ & $10.7 \mu \mathrm{b}$ & $11.87 \mu \mathrm{b}$ \\
$p_{t_{l}}>0.3 \mathrm{GeV},\left|y_{l}\right|<2.5$ & $2.62 \mu \mathrm{b}$ & $2.32 \mu \mathrm{b}$ & $2.47 \mu \mathrm{b}$ \\
$p_{t_{l}}>0.5 \mathrm{GeV},\left|y_{l}\right|<4.9$ & $0.89 \mu \mathrm{b}$ & $1.12 \mu \mathrm{b}$ & $0.97 \mu \mathrm{b}$ \\
$p_{t_{l}}>0.5 \mathrm{GeV},\left|y_{l}\right|<2.5$ & $0.285 \mu \mathrm{b}$ & $0.34 \mu \mathrm{b}$ & $0.288 \mu \mathrm{b}$ \\
$p_{t_{l}}>1 \mathrm{GeV}$ & $44.4 \mathrm{nb}$ & $47.5 \mathrm{nb}$ & $45.5 \mathrm{nb}$ \\
$p_{t_{l}}>1 \mathrm{GeV},\left|y_{l}\right|<4.9$ & $40.7 \mathrm{nb}$ & $43.6 \mathrm{nb}$ & $41.9 \mathrm{nb}$ \\
$p_{t_{l}}>1 \mathrm{GeV},\left|y_{l}\right|<2.5$ & $13.2 \mathrm{nb}$ & $18.1 \mathrm{nb}$ & $14.7 \mathrm{nb}$ \\
$p_{t_{l}}>1 \mathrm{GeV},\left|y_{l}\right|<1$ & $0.75 \mathrm{nb}$ & $0.98 \mathrm{nb}$ & $0.81 \mathrm{nb}$ \\
\hline \hline
\end{tabular}

momentum and rapidities of produced pairs in Table IV. Our results are consistent in themselves, and also consistent with other studies in the literature for multipair production [23]. Unlike the other studies in the literature, we also examined the double-muon pairs and simultaneous production of electronmuon pairs in our study.
[1] K. Kajantie, M. Kataja, L. McLerran, and P. V. Ruuskanen, Phys. Rev. D 34, 811 (1986); 34, 2746 (1986).

[2] C. R. Vane, S. Datz, P. F. Dittner, H. F. Krause, C. Bottcher, M. R. Strayer, R. Schuch, H. Gao, and R. Hutton, Phys. Rev. Lett. 69, 1911 (1992).

[3] C. Bottcher and M. R. Strayer, Phys. Rev. D 39, 1330 (1989).

[4] C. A. Bertulani and G. Baur, Phys. Rep. 163, 299 (1988).

[5] M. C. Güçlü, J. C. Wells, A. S. Umar, M. R. Strayer, and D. J. Ernst, Phys. Rev. A 51, 1836 (1995).

[6] M. C. Güçlü, J. Li, A. S. Umar, D. J. Ernst, and M. R. Strayer, Ann. Phys. (NY) 272, 7 (1999).

[7] E. Fermi, Z. Phys. 29, 315 (1924).

[8] C. F. von Weizsäcker, Z. Phys, 88, 612 (1934); E. J. Williams, Phys. Rev. 45, 729 (1934).

[9] A. Alscher, K. Hencken, D. Trautmann, and G. Baur, Phys. Rev. A 55, 396 (1997).

[10] G. Baur, K. Hencken, A. Aste, D. Trautmann, and S. R. Klein, Nucl. Phys. A 729, 787 (2003).

[11] ATLAS Collaboration et al., ATLAS-CONF-2016-025 (2016).

[12] E. Abbas et al., Eur. Phys. J. C 73, 2617 (2013).

[13] J. Adam et al. (ALICE Collaboration), Phys. Rev. Lett. 116, 222301 (2016).

[14] M. Aboud et al. (ATLAS collaboration), Phys. Rev. Lett. 121, 212301 (2018).

[15] M. Dyndal et al. (ATLAS Collaboration), Nucl. Phys. A 967, 281 (2017).

[16] J. Adam et al. (STAR Collaboration), Phys. Rev. Lett. 121, 132301 (2018).
[17] L. Adamczyk et al. (STAR Collaboration), Phys. Lett. B 770, 451 (2017).

[18] S. Afanasiev et al. (PHENIX Collaboration), Phys. Lett. B 679, 321 (2009).

[19] M. Y. Sengul, M. C. Güçlü, O. Mercan, and N. G. Karakus, Eur. Phys. J. C 76, 428 (2016).

[20] M. C. Güçlü, Nuc. Phys. A 668, 149 (2000).

[21] E. Kurban and M. C. Güçlü, Phys. Rev. C 96, 044913 (2017).

[22] S. Klein and J. Nystrand, The STARLIGHT website, http:// starlight.hepforge.org.

[23] M. Kłusek-Gawenda and A. Szczurek, Phys. Lett. B 763, 416 (2016).

[24] A. van Hameren, M. Kłusek-Gawenda, and A. Szczurek, Phys. Lett. B 776, 84 (2018).

[25] C. Azevedo, V. P. Gonçalves, and B. D. Moreira, Eur. Phys. J. C 79, 432 (2019).

[26] S. R. Klein, Phys. Rev. C 97, 054903 (2018).

[27] S. Klein, A. H. Mueller, Bo-Wen Xiao, and F. Yuan, Phys. Rev. Lett. 122, 132301 (2019).

[28] M. Kłusek-Gawenda, R. Rapp, W. Schäfer, and A. Szczurek, Phys. Lett. B 790, 339 (2019).

[29] W. Zha, L. Ruan, Z. Tang, Z. Xu, and S. Yang, Phys. Lett. B 781, 182 (2018).

[30] A. Szczurek, Nucl. Phys. B 219-220, 17 (2011).

[31] C. R. Vane, S. Datz, P. F. Dittner, H. F. Krause, R. Schuch, H. Gao, and R. Hutton, Phys. Rev. A 50, 2313 (1994).

[32] C. R. Vane, S. Datz, E. F. Deveney, P. F. Dittner, H. F. Krause, R. Schuch, H. Gao, and R. Hutton, Phys. Rev. A 56, 3682 (1997). 